Mots. Les langages du politique

$114 \mid 2017$

Le rapport, entre description et recommandation

\title{
Josiane Boutet, Le pouvoir des mots
}

Paris, La Dispute, 2016, 256 p.

\section{Émilie Devriendt}

\section{(2) OpenEdition}

\section{Journals}

Édition électronique

URL : https://journals.openedition.org/mots/22873

DOI : $10.4000 /$ mots. 22873

ISSN : 1960-6001

Éditeur

ENS Éditions

\section{Édition imprimée}

Date de publication : 10 juillet 2017

Pagination : 210-216

ISSN : 0243-6450

Référence électronique

Émilie Devriendt, " Josiane Boutet, Le pouvoir des mots », Mots. Les langages du politique [En ligne], 114 2017, mis en ligne le 20 juin 2017, consulté le 22 avril 2022. URL : http://journals.openedition.org/ mots/22873; DOI : https://doi.org/10.4000/mots.22873

Ce document a été généré automatiquement le 22 avril 2022

(C) ENS Éditions 


\title{
Josiane Boutet, Le pouvoir des mots
}

\author{
Paris, La Dispute, 2016, 256 p.
}

\section{Émilie Devriendt}

\section{RÉFÉRENCE}

BOUTET J., Le pouvoir des mots. Paris, La Dispute, 2016, 256 p. (nouv. éd.)

1 La sociolinguiste Josiane Boutet, pionnière des études du langage au travail, livre une nouvelle édition augmentée et entièrement remaniée d'un ouvrage initialement paru en 2010. Les exemples, empruntés à différents domaines (politique, médiatique, publicitaire, militant, scientifique, juridique, militaire, religieux...), à des époques et à des sociétés variées, mais en particulier à la France contemporaine, ont été mis à jour jusqu'en septembre 2015, et contribuent ainsi à renforcer l'actualité de l'ouvrage, et, plus largement, «son ancrage dans les préoccupations, les intérêts, les questionnements de notre société » (p.12). Josiane Boutet propose en effet un «livre politique de linguiste». En l'adressant aux «citoyens confrontés dans leur vie quotidienne et professionnelle aux usages et mésusages des mots, à l'efficacité des discours, politiques ou autres » (p. 13) autant qu'aux linguistes, l'auteure entend « faire une linguistique "dans le monde" ( (Labov, 1989) : confronter la linguistique avec les faits sociaux, non seulement pour mesurer la pertinence d'une théorie, mais aussi pour participer par la recherche en sciences humaines et sociales d'une démarche de transformation du réel.

2 Organisée en six parties, la nouvelle édition propose une progression de lecture qui met en valeur la thèse de l'ouvrage. Cette thèse, illustrée dès l'exergue emprunté à l'anthropologue Bronislaw Malinowski, est exposée dans une première partie théorique, inédite, où l'auteure présente sa «conception actionnelle du langage envisagé centralement comme une pratique sociale» (p.9), et synthétisée dans une conclusion elle aussi inédite : «[l]es mots sont loin de fonctionner comme de simples vecteurs de la communication, comme le pensent les conceptions technicistes et instrumentales de la communication verbale qui voudraient les réduire à un pur 
transfert d'informations entre des êtres libres, conscients et égaux.»(p. 252-253) Chaque partie déroule ce fil conducteur, constituée de chapitres dont le titre est structuré en deux temps : une citation qui sera, parmi d'autres exemples, soumise à l'analyse linguistique, et la dénomination de la « pratique langagière » qu'à travers elle l'auteure a choisi d'illustrer.

3 La première partie ("De la communication à l'action. Les pratiques langagières ») s'attache à contredire l'«idéologie linguistique de la neutralité instrumentale » du langage, caractéristique des sociétés capitalistes contemporaines, et que J. Boutet illustre en empruntant des exemples à la communication politique ou aux doctrines managériales. Après un retour attendu sur les travaux de Roman Jakobson consacrés aux différentes fonctions du langage, prolongés par la modalisation des situations de communication chez Dell Hymes, mais initiés par les recherches d'un autre ethnographe, B. Malinowski, référence majeure de cette nouvelle édition, l'auteure développe un aspect abordé plus tardivement en linguistique : celui de la "puissance d'action du langage sur le monde» (ou performativité), pour évoquer les années 1980 comme «tournant praxéologique » des sciences humaines et sociales, où s'inscrivent ses propres travaux. De ces derniers elle propose une contextualisation éclairant le concept central de pratiques langagières, élaboré avec Pierre Fiala et Jenny Simonin en 1976, soulignant ce qu'il devait alors au contexte politique et culturel ayant succédé à Mai 1968, ainsi que la filiation discursive du néologisme, dans la référence au Marx des Thèses sur Feuerbach (1845), diluée aujourd'hui, mais que l'ouvrage de J. Boutet entend revivifier, en illustrant l'actualité et la nécessité des perspectives critiques ouvertes par une conception du langage (et du travail) comme "praxis fondamentale(s) de transformation du monde par les êtres humains » (p. 49).

4 La deuxième partie («Les combats politiques sont aussi des combats sur les mots») aborde trois pratiques langagières illustrant la «construction sociale du sens » théorisée dans les travaux antérieurs de J. Boutet: redéfinition ou appropriation de mots-enjeux selon un point de vue de classe, "nazification" de la langue sous le troisième Reich, entreprises totalitaires de transformation de la langue (le novlangue d'Orwell) - ces deux dernières illustrant de manière extrême l'appropriation d'un bien commun par un groupe dominant, mais aussi le "pouvoir d'action propre des langues sur les consciences et la pensée » (p. 75).

5 La troisième partie ("Le pouvoir de nommer») comprend trois chapitres rendant compte de trois dimensions actionnelles de l'acte de nommer: catégorisations de pseudo-objets («le Grand Remplacement» de l'idéologue d'extrême droite Renaud Camus) et de groupes sociaux (homosexuels, "migrants », ouvriers) et leurs effets sur les représentations collectives; "programmes d'action » engagés par les «mots du travail » associant certaines pratiques langagières (euphémisation ou hyperbole) à une conduite magique (caillou pour un bloc de pierre de plusieurs tonnes, flingueuse pour le bloc opératoire); réappropriation et réévaluation positive, par des groupes minoritaires ou dominés, de désignants stigmatisants au regard de la norme dominante.

6 Avec la quatrième partie ("Techniques de l'efficacité verbale »), ce sont l'organisation et les fonctions des discours qui font l'objet des exemples analysés. Les deux premiers chapitres explorent la tradition rhétorique en analysant la performativité de certains énoncés politiques, la force des discours publicitaires, mais aussi les préconisations managériales en usage dans les centres d'appel (la «voix du sourire»). Les deux 
derniers sont consacrés à la créativité verbale, individuelle ou collective, où l'auteure, à travers des analyses fines de la forme des énoncés (enseignes, slogans, insultes rituelles), entend illustrer des cas où la «fonction poétique» prend le pas sur la fonction référentielle du langage (Jakobson).

7 La cinquième partie ( Les actes de langage ») revient sur le débat sur l'origine de la puissance performative de la langue ayant opposé Bourdieu et les tenants de l'approche pragmatiste anglo-saxonne (Austin, Searle). Chacun des trois chapitres est consacré à un acte: "jurer», «ordonner», «injurier». Les exemples analysés concernent principalement les sociétés occidentales contemporaines (discours politiques, échanges oraux militaires strictement réglementés, formes de commandement en situation professionnelle, langage d'autorité du droit), et viennent illustrer le postulat de «transformations majeures dans les conditions d'énonciation et de réception des discours d'autorité » (p.184), associé à celui d'une «informalisation progressive des relations sociales » (p. 205).

8 La sixième partie ("La puissance magique des mots») emprunte une voie moins fréquentée par les linguistes et s'appuie sur des travaux d'ethnographie de la communication pour aborder en détail les « effets des mots dans le monde réel ». Qu'elle évoque leur puissance symbolique ou leur rapport avec l'inconscient, il s'agit d'illustrer les relations étroites entre langage humain et processus psychiques. Dans son chapitre consacré au récit d'un "jeune öm sqizofrène», J. Boutet analyse une situation individuelle où le pouvoir des mots tient à leur forme sonore, révélant une fonction (ici, de blessure psychique) comparable selon elle à une forme de sorcellerie. Le chapitre suivant présente cette pratique sociale comme exemple propre à «questionner les conceptions technocratiques de la langue ». L'auteure se tourne vers les caractéristiques formelles de l'énonciation des paroles magiques (modalités de voix, style communicationnel, formes plus ou moins figées), pour mieux faire le lien avec des pratiques sociales contemporaines apparemment banales, pouvant néanmoins relever de cette dimension magique (mots tabous, prières profanes).

La conclusion complète et prolonge la présentation du modèle de Malinowski, évoquant des discours organisés «selon une sorte de continuum, depuis les paroles de la magie jusqu'à celles de la science ", deux pôles dont "le poids, l'importance sociale et la valeur [...] sont sous la dépendance des cultures et des histoires de chaque société humaine particulière » (p. 248). J. Boutet revient aussi sur la dimension sociale de toute pratique langagière envisagée comme « tension constitutive entre le caractère subjectif de l'activité de langage et le caractère collectif et arbitraire des langues » (p. 250), tension à laquelle les locuteurs d'une langue, comme acteurs sociaux, peuvent réagir de différentes manières en fonction des situations sociales. Ainsi, les situations ordinaires sont davantage propices à l'intégration et à la reproduction des normes dominantes (" on accepte les mots que la langue nous propose et nous impose », ibid.), mais les situations quotidiennes appellent parfois un travail sur les mots, en particulier lorsque leur inadéquation révèle un antagonisme social. Enfin, comme le suggèrent les témoignages de survivantes et survivants des camps d'extermination, les enjeux et difficultés de la nomination culminent dans des situations extrêmes : « des conditions de vie inouïes, des situations sociales encore impensées doivent générer de nouveaux mots pour les exprimer » (p. 251).

En présentant ce travail comme le fruit de choix politiques assumés, J. Boutet prend parti dans la réflexion menée ces dernières années autour de l'« engagement du 
chercheur » en sciences du langage (Koren, 2013), bien que son positionnement ne soit pas explicitement envisagé dans sa dimension éthique. Elle élabore son intervention "politique de linguiste " relative à la puissance agissante des mots à la fois comme entreprise critique de démystification de leurs "usages et mésusages ", et comme description de leur puissance émancipatrice. Par la réflexion qui le traverse s'agissant de la notion d'autorité, l'ouvrage tend à illustrer la «continuité naturelle entre l'analyse des pouvoirs du discours et la critique des discours du pouvoir» (Maingueneau, 2014, p. 58).

11 La dimension critique est principalement associée à l'analyse de discours politiques institutionnels, en particulier ceux de la droite et de l'extrême droite contemporaines, ainsi qu'aux discours totalitaires passés : dans tous les cas, il s'agit d'objets à même d'appeler une écriture militante, comme l'a souligné Émilie Née (2016) à propos d'un autre ouvrage "grand public" à visée démystificatrice ${ }^{1}$. Initialement paru sous le mandat de Nicolas Sarkozy, alors perçu comme emblématique des mutations contemporaines du discours politique ${ }^{2}$, l'ouvrage de J. Boutet propose un éclairage propre, entre autres, de ce phénomène que semble avoir confirmé le mandat de François Hollande (Mayaffre, 2016). Le discours politique illustre par ailleurs, de manière « évidente lorsqu'il s'agit du discours présidentiel » (Mayaffre, 2012, p. 18), la double dimension du langage, référentielle et actionnelle ou performative, analysée dans l'ouvrage de J. Boutet. L'illustration de couverture de la nouvelle édition, croquis de Jaurès " pour servir à illustrer l'histoire de l'éloquence ", vient encore renforcer cet aspect et, peut-être, évoquer l'échéance l'élection présidentielle 2017 - l'« horizon collectif» étant plus généralement associé par J. Boutet à l'idée de "menace», récurrente dans l'ouvrage s'agissant des entreprises totalitaires d'appropriation du bien commun. Si l'analogie n'est jamais explicitée ${ }^{3}$, la part faite à l'analyse des novlangues ou « langues de bois » associées à ces régimes totalitaires tend à signifier la nécessité politique de combattre les « conceptions technicistes et instrumentales » du langage dans leur version managériale-néolibérale contemporaine, d'où qu'elles émanent. Pour ce faire, mais sans jamais prétendre que la réalité se réduirait aux discours («Les combats politiques sont aussi des combats sur les mots»-je souligne)" la nouvelle édition renforce son argumentation relative à l'efficacité symbolique des mots, prenant le risque d'avancer sur une ligne de crête, en particulier dans sa dernière partie.

12 Cet ouvrage se présente aussi comme défense et illustration de la créativité et de la puissance d'agir que les discours sont à même de conférer aux acteurs sociaux, dans des situations ordinaires ou extraordinaires, et en particulier dans les luttes sociales, comme en témoignent les exemples relevant de l'histoire et de l'actualité du mouvement ouvrier ou des «nouveaux mouvements sociaux». Ce faisant, il rejoint également l'éthique d'une analyse du discours envisagée comme "propre à rendre compte de la capacité d'action (agency) des individus à l'horizon de l'émancipation humaine $»^{5}$. Sa réflexion sur la langue comme bien commun fait aussi écho aux travaux de Jacques Rancière ${ }^{6}$, pour qui le pouvoir des mots tient à leur puissance de dissensus dès lors qu'ils sont proférés sur la scène publique par celles et ceux qui n'étaient pas les détenteurs autorisés du sens (Rancière, 2009). Cette prise de parole consiste à réintroduire du commun sous la forme du dissensus, un tel pouvoir se voyant contesté «par les maîtres des désignations et des classifications, lesquels, pour révoquer ce pouvoir, nient purement et simplement cette capacité parlante » (Ibid., p. 175). Cette mise en question de l'autorité "légitime», en introduisant la possibilité d'un 
changement social, a apporté un éclairage important dans le débat sur la nature de la performativité7. Elle invite ici à réinterpréter le "pouvoir des mots » comme " pouvoir de parler ${ }^{8}$, selon l'expression de Michel de Certeau dans un écrit consacré à Mai 68 (Certeau, 1994, p. 66 sq.), ce «festival de la parole agissante» (Viart, 2008, p. 15), dont l'expérience fut constitutive de nombreux parcours de recherche intéressés aux rapports entre langage et société .

Les représentations propres aux acteurs sociaux eux-mêmes s'agissant du pouvoir magique des mots tiennent une place prépondérante dans l'ouvrage de J. Boutet, notamment à travers les travaux ethnographiques cités, qui en décrivent les effets sans séparer ces représentations de leur base matérielle. Relativement à cet aspect, on pourra toutefois regretter dans l'ouvrage l'absence de toute référence aux questionnements poétiques ${ }^{10}$, dans la mesure où bon nombre ont aussi concerné les pratiques et conceptions du langage dans les sociétés dites primitives, pour souligner leur pouvoir d'action sur le réel : c'est par exemple le cas des recherches Surréalistes sur la fonction des mythes (Péret, 1965 [1945]). Plus près de nous, on trouverait une réflexion proche des préoccupations de J. Boutet dans l'essai du poète Daniel Blanchard (2012), ancien membre du groupe Socialisme ou barbarie, lequel définit la poésie comme "l'essence même» du langage, et voit dans sa méconnaissance ou sa dénégation contemporaine «l'injonction qui nous est faite d'enfermer très étroitement le langage dans une fonction instrumentale [...] et d'arrêter notre pensée à la matérialité des faits supposés établis, afin de nous aveugler sur l'illimité de nous-mêmes comme du monde " (Ibid., p. 49), dans une société où le code aurait pris le pas sur la langue. Il n'est pas anodin que certaines de ces réflexions tiennent pour centrale la question des rapports entre poésie et révolution - non pas d'une poésie au service de la révolution, mais comme partie prenante d'une transformation radicale de l'existant. C'est le sens de cet extrait de B. Péret (Ibid.), "poète donc révolutionnaire ", qui pourra également faire écho au continuum des deux pôles du modèle de Malinowski mentionnés par J. Boutet :

Celui qui la comprend [l'unité de la pensée] se révèle un révolutionnaire qui tend, peut-être à son insu, à rejoindre la poésie. Il s'agit en effet de réduire une fois pour toutes l'opposition artificielle créée par des esprits sectaires venus de l'un et l'autre côtés de la barricade qu'ils ont élevée de concert, entre la pensée poétique, qualifiée pré-logique, et la pensée logique, entre la pensée rationnelle et l'irrationnel.

\section{BIBLIOGRAPHIE}

BLANCHARD Daniel, 2012, Crise de mots, Paris, Éd. du Sandre.

CALVET Louis-Jean et VÉRonis Jean, 2008, Les mots de Nicolas Sarkozy, Paris, Éd. du Seuil.

CERTEAU Michel de, 1994 [1968], La prise de parole et autres écrits politiques, Paris, Éd. du Seuil.

CLASTRES Hélène, 2011, « De quoi parlent les Indiens », Cahier Pierre Clastres, M. Abensour et A.

Kupiec éd., Paris, Sens \& Tonka, p. 211-220.

Clastres Pierre, 1974, La Société contre l'État, Paris, Éd. du Seuil. 
GUILHAUMOU Jacques et MAZIÈRES Francine, 2010, « “Ainsi, nous sommes qui nous sommes dans le Mississippi” ", Semen, n 29, <http://semen.revues.org/8782> (consulté le 4 avril 2017).

GUILHAUMOU Jacques, 2013, « L'engagement d'un historien du discours : trajet et perspectives », Argumentation et analyse du discours, $\mathrm{n}^{\circ}$ 11, <http://aad.revues.org/1599> (consulté le 4 avril 2017). HAZAN Éric, 2006, LQR. La propagande au quotidien, Paris, Éd. Raisons d'agir.

KOREN Roselyne éd., 2013, « Analyses du discours et engagement du chercheur », Argumentation et analyse du discours, $\mathrm{n}^{\circ}$ 11, <http://aad.revues.org/1515> (consulté le 4 avril 2017).

LABOV William, 1989, « La théorie linguistique à l'épreuve de la justice ", Actes de la recherche en sciences sociales, $\mathrm{n}^{\circ}$ 76-77, p. 104-114.

MAINGUENEAU Dominique, 2014, Discours et analyse du discours, Paris, A. Colin.

MAYAFFRE Damon, 2012, Nicolas Sarkozy. Mesure et démesure du discours. 2007-2012, Paris, Presses de la Fondation nationale des sciences politiques.

MAYAFFRE Damon, 2016, « Du candidat au président. Panorama logométrique de François

Hollande ", Mots. Les langages du politique, $\mathrm{n}^{\circ} 112$, p. 81-92.

NÉE Émilie, « [Compte rendu de lecture de] Cécile Alduy et Stéphane Wahnich, Marine Le Pen prise aux mots, Paris, Éd. du Seuil, 2015 », Mots. Les langages du politique, nº 110, p. 187-192.

PÉRET Benjamin, 1965 [1945], « La parole est à Péret », Le déshonneur des poètes précédé de La parole est à Péret, Paris, J.-J. Pauvert.

RANCIÈRE Jacques, 2009 [2000], « Les mots du dissensus » (entretien avec Davide Panagia), Et tant pis pour les gens fatigués. Entretiens, Paris, Éd. Amsterdam.

VIART Dominique, 2008, « Les héritages de Mai 68 », Écrire, mai 68, C. Flohic éd., Paris, Argol, p. 9-29.

\section{NOTES}

1. Ouvrage par ailleurs contesté. J. Boutet le cite p. 67.

2. Louis-Jean Calvet et Jean Véronis (2008) - cités par J. Boutet, p. 66 - parlaient à son propos d'inauguration d'«un langage nouveau en politique». Damon Mayaffre (2012) évoquera un «basculement du discours présidentiel».

3. Contrairement à certains ouvrages de lexicographie populaire contemporains (Hazan, 2006).

4. Écueil fréquemment pointé à propos du «tournant linguistique » des sciences humaines et sociales, et / ou des approches postmodernes.

5. J. Guilhaumou (2013, § 16). J. Boutet cite d'autres travaux de J. Guilhaumou, p. 51 et p. 113.

6. Cité p. 58-60, principalement comme source des écrits ouvriers reproduits et analysés par J. Boutet.

7. Les travaux de l'anthropologue Pierre Clastres consacrés à la culture Tupi-Guarani, par la réflexion menée sur les rapports entre langage et pouvoir, peuvent également servir à éclairer les questions posées par ce débat: dans les terrains qu'il a décrits comme "sociétés contre l'État ", les figures de l'autorité ont le monopole des discours, mais y sont aussi cantonnées, ces discours n'ayant aucun pouvoir politique (typiquement, le chefs ne peuvent donner d'ordres), leur unique fonction étant de célébrer les traditions (Clastres, 1974, chap. 7 et passim). Sur la question du langage dans l'œuvre de P. Clastres, voir Clastres 2011.

8. Littéralement, de prendre la parole à qui se l'était appropriée. 
9. Par exemple J. Guilhaumou et F. Mazière (2010), J. Guilhaumou (2013), J. Rancière (2009). J. Boutet évoque cette période p. 43.

10. À l'exception de la courte section consacrée au travail d'Apollinaire sur les adjectifs, analysé comme forme de volontarisme linguistique.

\section{AUTEURS}

\section{ÉMILIE DEVRIENDT}

Université de Toulon, Babel, EA 2649 